\title{
Laser heat treatment of metals using rotating and dithering beams
}

\author{
M. Sistaninia \\ Computational Materials Laboratory \\ Ecole Polytechnique Fédérale de Lausanne
}

\section{Introduction}

Laser heating involves the use of high intensity laser radiation to rapidly heat the surface of metals for melting, forming or hardening. For some of the laser heat treatment of metals, it is preferable to use a wider laser beam (larger size of beam spot) with a uniform heat intensity distribution, e.g. for laser surface hardening in order to attain a more uniform hardened depth, a wide and uniform laser beam is needed. This can be accomplished by the following three approaches (Luxon, 1984):

The first one is achieved by rotating the beam optically, thereby producing an overlapping spiral track or by dithering the beam (rocking the lens or mirror) perpendicular to the track, thus producing a zigzag pattern. Figure 1 is an illustration of these techniques. The second alternative involves the use of a bimodal (TEM11) shaped laser beam. Unfortunately it is difficult to maintain a high-quality, high-order mode over a long period of time. The third alternative involves the use of not-so-sharp focused high-peak power, low order (TEM00) mode Gaussian laser beam.

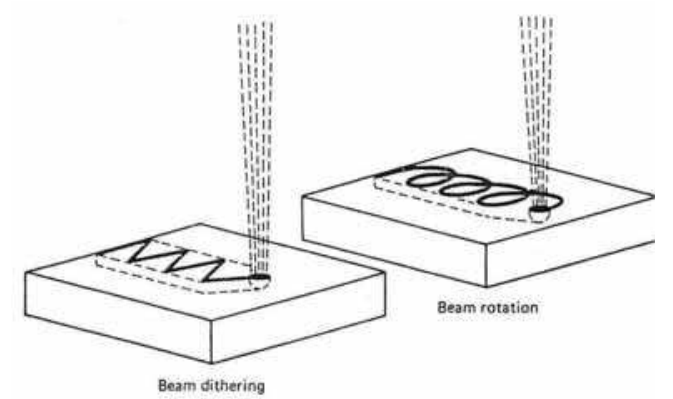

Fig. 1. Active methods for spreading out a laser beam for surface treatment applications

The first theoretical and experimental investigations of laser heating of materials dated back to 1960s. Laser heating, although possible for a number of years, is a technology which is still in its infancy. Modeling (analytically or numerically) the thermal field due to laser heating has been subject to many researches. Some of these works were carried out on 
quasi-steady state, e.g. (Rajadhyaksha \& Michaleris, 2000). In the other works a transient model were developed to model the thermal field in laser heating, e.g. (Komanduri \& Hou, 2004). Numerical integration, finite difference, and finite element have all been successful in predicting thermal field of laser heating metals for different laser beam distributions.

In analytical thermal models it is assumed that the thermal properties of materials are constant. However the thermal properties of materials change a lot with temperature, because of that the numerical results agree better than the analytical results with the experimental results. The analytical models are usually used to improve the results of the numerical models by finding the parameters of the numerical model properly.

In this chapter the thermal fields during laser heating with rotating beam (LHR) and laser heating with dithering beam (LHD) are modeled by employing both three-dimensional analytical model and finite element model. In the finite element model the laser beam is considered as a moving plane heat flux to establish the temperature rise distribution in the work-piece, while in the analytical model laser beam is considered as an internal heat generation.

At the second part of this chapter the thermal fields of LHR and LHD are used to predict the hardened region of laser hardening with rotating and dithering beams. In this chapter also the advantages of using rotating and dithering beams for laser forming of plates are explored. The temperature distribution from the thermal modeling is input as a body load for a three-dimensional nonlinear structural analysis to predict the distortions of laser forming with dithering and rotating beams.

Since all these simulations are according to uncoupled thermoelasticity, at the last part of this chapter a study is done to see the accuracy of these simulations. Thermoelastic temperature, displacement and stress in heat transfer during laser surface hardening are solved in both Lagrangian and Eulerian formulations of coupled thermoelectricity.

\section{Mathematical modeling of temperature during LHR and LHD}

In this section, the transient thermal field in LHR and LHD are modeled by employing both three-dimensional analytical model and three-dimensional numerical model. For LHR and LHD even if the heat flux is fixed in space and the work-piece moves (Eulerian formulation) the thermal field remains transient and doesn't reduce to a steady-state problem, hence the thermal modeling of LHR and LHD needs a Lagrangian system with transient formulation. In a typical Lagrangian system, both the reference and the material configuration are fixed in space and all the spatial parameters are calculated with respect to the fixed reference configuration.

\subsection{Analytical model}

An analytical model, based on the well-known transient heat conduction equation, is adopted to describe the time and space temperature distribution $\mathrm{T}(\mathrm{r}, \mathrm{t})$ in the material under the action of a laser beam that may dithering or rotating:

$$
\rho c_{P} \frac{\partial T}{\partial t}+\nabla(-K \nabla T)=f(r, t)
$$


where $c_{p}, \rho$ and $K$ denote, respectively, the specific heat, density and thermal conductivity of the work-piece and the heat source term $f(r, t)$, at the right side of the Eq. (1), is identified as the heat distribution of the laser beam.

If $c_{p}, \rho$ and $K$ are temperature independent, Eq. (1) is reduced to:

$$
\frac{1}{\alpha} \frac{\partial T}{\partial t}-\nabla^{2} T=\frac{f(r, t)}{K}
$$

where $\alpha=K / \rho c_{p}$, is the thermal diffusivity. In the case of three-dimensional transient, nonhomogeneous heat conduction problem given by Eq. (2), the solution for $T(r, t)$ can be expressed in term of the three-dimensional Green's function (Farrahi \& Sistaninia, 2009),

$$
T(\mathbf{r}, t)=\frac{\alpha}{K} \int_{\tau=0}^{t} d \tau \int_{R} G\left(\mathbf{r}, t \mid \mathbf{r}^{\prime}, \tau\right) f\left(\mathbf{r}^{\prime}, \tau\right) d v^{\prime}+\left.\int_{R} G\left(\mathbf{r}, t \mid \mathbf{r}^{\prime}, \tau\right)\right|_{\tau=0} F\left(\mathbf{r}^{\prime}\right) d v^{\prime}
$$

where $F\left(\mathbf{r}^{\prime}\right)$ is the initial temperature distribution. A Green's function is an integral kernel that can be used to solve an inhomogeneous differential equation with boundary conditions. It plays an important role in the solution of linear ordinary and partial differential equations, and is a key component to the development of boundary integral equation methods. For an arbitrary linear differential operator $\bar{L}$ in three dimensions, the Green's function $G\left(\mathbf{r}, \mathbf{r}^{\prime}\right)$ is defined by analogy with the one-dimensional case by,

$$
\bar{L} G\left(\mathbf{r}, t \mid \mathbf{r}^{\prime}, \tau\right)=\delta\left(\mathbf{r}-\mathbf{r}^{\prime}\right)
$$

For the Eq. (3) the three-dimensional Green's function in rectangular coordinates can be obtained from the product of three one-dimensional Green's function as:

$$
G\left(x, y, z, t \mid x^{\prime}, y^{\prime}, z^{\prime}, \tau\right)=G 1\left(x, t \mid x^{\prime}, \tau\right) \times G 2\left(y, t \mid y^{\prime}, \tau\right) \times G 3\left(z, t \mid z^{\prime}, \tau\right)
$$

For this problem $G\left(x, y, z, t \mid x^{\prime}, y^{\prime}, z^{\prime}, \tau\right)$ denotes the temperature at point $(x, y, z)$ at time $t$ due to a point source at point $\left(x^{\prime}, y^{\prime}, z^{\prime}\right)$ at time $\tau$ with the intensity distribution of $f\left(x^{\prime}, y^{\prime}, z^{\prime}, \tau\right)$. Since only at $\mathrm{z}^{\prime}=0 ; \quad f\left(x^{\prime}, y^{\prime}, z^{\prime}, \tau\right) \neq 0$, the three-dimensional Green's function, $G\left(x, y, z, t \mid x^{\prime}, y^{\prime}, z^{\prime}=0, \tau\right)$ should be found. The one-dimensional Green's functions G1, G2 and G3 in Eq. (5) depend on the extent of the region (i.e., finite, semi infinite or infinite) and the boundary conditions. In table (1) the one dimensional Green's functions for different regions and boundary conditions are presented from Refs. (Polyanin, 2002) and (Me Sistaninia et al., 2009). 


\begin{tabular}{|l|l|l|l|}
\hline region & $\begin{array}{l}\text { Boundary } \\
\text { condition }\end{array}$ & Equation & \\
\hline Infinite & ---- & $G\left(s, t \mid s^{\prime}, \tau\right)=[4 \pi \alpha(t-\tau)]^{-1 / 2} \exp \left(-\frac{\left(s-s^{\prime}\right)^{2}}{4 \alpha(t-\tau)}\right)$ & $(6)$ \\
\hline $\begin{array}{l}\text { Semi- } \\
\text { infinite }\end{array}$ & insulated & $G\left(s, t \mid s^{\prime}, \tau\right)=[4 \pi \alpha(t-\tau)]^{-1 / 2}\left[\exp \left(-\frac{\left(s-s^{\prime}\right)^{2}}{4 \alpha(t-\tau)}\right)+\exp \left(-\frac{\left(s+s^{\prime}\right)^{2}}{4 \alpha(t-\tau)}\right)\right]$ & $(7)$ \\
\hline $\begin{array}{l}\text { Semi- } \\
\text { infinite }\end{array}$ & convection & $G\left(s, t \mid s^{\prime}=0, \tau\right)=\int_{\beta=0}^{\infty}\left(e^{-\alpha \beta^{2}(t-\tau)} \frac{2}{\pi} \frac{\beta}{\beta^{2}+H^{2}}\right.$ & $(8)$ \\
\hline Finite & insulated & $(\beta \cos \beta s+H \sin \beta s) d \beta$ & $(9)$ \\
\hline Finite & convection & $G\left(s, t \mid s^{\prime}=0, \tau\right)=\frac{1}{L}+\frac{2}{L} \sum_{n=1}^{\infty} e^{-\alpha\left(\frac{n \pi}{L}\right)^{2}(t-\tau)} \cos \left(\frac{n \pi}{L} s\right)$ & $(10)$ \\
\hline & {$\left[\left(\beta_{m}^{2}+H_{1}^{2}\right)\left(L+\frac{H_{2}}{\beta_{m}^{2}+H_{2}^{2}}\right)+H_{1}\right]^{-1}\left(\beta_{m} \cos \beta_{m} s+H_{1} \sin \beta_{m} s\right)$} & \\
\hline
\end{tabular}

Table 1. One-dimensional Green's functions

For example for a semi infinite medium with isolated boundary condition (along $\mathrm{Z}$ direction) the three-dimensional Green's function can be found by substitutions $G 1\left(x, t \mid x^{\prime}, \tau\right)$ and $G 2\left(y, t \mid y^{\prime}, \tau\right)$ from Eq. (6) and $G 3\left(z, t \mid z^{\prime}, \tau\right)$ from Eq. (7), in Eq. (5),

$$
G\left(x, y, z, t \mid x^{\prime}, y^{\prime}, z^{\prime}=0, \tau\right)=2[4 \pi \alpha(t-\tau)]^{-1.5} \exp \left(-\frac{\left(x-x^{\prime}\right)^{2}+\left(y-y^{\prime}\right)^{2}+z^{2}}{4 \alpha(t-\tau)}\right)
$$

The temperature distribution for the semi infinite medium could be obtained by substitution $G\left(\mathbf{r}, t \mid \mathbf{r}^{\prime}, \tau\right)$ from Eq. (11), in Eq. (3). It is easier to calculate the integration Eq. (3) in polar coordinate with substitutions $x^{\prime}=x-r \cos \theta, y^{\prime}=y-r \sin \theta$ as,

$$
\begin{aligned}
& T(x, y, z, t)=T_{o}+\frac{2 \alpha}{K} \int_{0}^{t} \int_{-\pi}^{\pi} \int_{0}^{\infty}[ \\
& \left.f(x-r \cos \theta, y-r \sin \theta, \tau)[4 \pi \alpha(t-\tau)]^{-1.5} \exp \left(-\frac{r^{2}+z^{2}}{4 \alpha(t-\tau)}\right) r\right] d r d \theta d \tau
\end{aligned}
$$

In this equation, $f(x-r \cos \theta, y-r \sin \theta, \tau)$ is the beam intensity distribution, which for a Gaussian heat intensity distribution that moves along a straight line is obtained as, 


$$
f(x-r \cos \theta, y-r \sin \theta, \tau)=\frac{3 Q}{\pi r_{o}^{2}} \exp \left\{-3\left(\frac{(x--r \cos \theta-v \tau)^{2}+(y-r \sin \theta)^{2}}{r_{o}^{2}}\right)\right\}
$$

where $r_{0}$ is the characteristic radius, defined as the radius at which the intensity of the laser beam falls to $5 \%$ of the maximum intensity, $Q$ is the power transferred into the substrate (equal to $A \times P$, where $\mathrm{A}$ is the absorptivity and $\mathrm{P}$ is the laser power) and $v$ is the laser velocity along moving line (longitudinal velocity).

The thickness of the work-pieces used for laser heat treatment is usually enough to model it as a semi infinite medium. In Figure 2 the effects of thickness on the temperature history of a point on the heated surface is shown. As you see the thickness of the work-piece for thicknesses bigger than the diameter of the laser spot $(3 \mathrm{~mm})$ dose not affect the temperature profile. Hence for this case the work-pieces with thickness bigger than $3 \mathrm{~mm}$ can be modeled as a semi infinite medium.

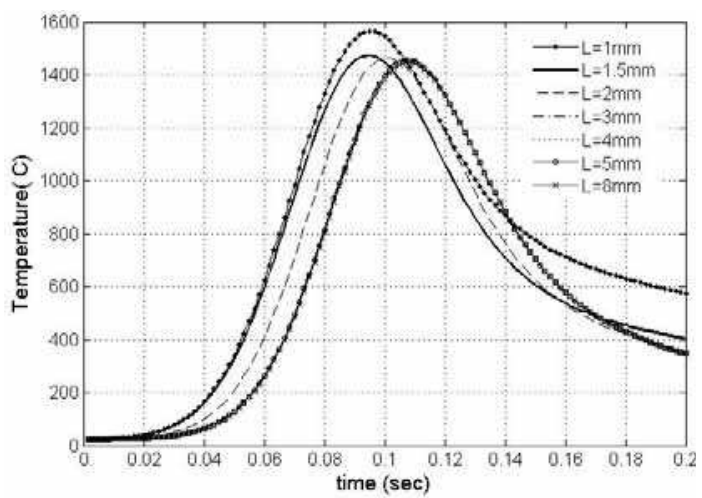

Fig. 2. Temperature history of a Gaussian beam mode of a work piece with different thickness (characteristic radius of heat flux $r_{0}=3 \mathrm{~mm}$, Power $Q=340 \mathrm{~W}$ and velocity $v=30 \mathrm{~mm} / \mathrm{s}$ )

For a dithering beam $f(x-r \cos \theta, y-r \sin \theta, \tau)$ is obtained as (Farrahi \& Sistaninia, 2009),

$$
f(x-r \cos \theta, y-r \sin \theta, \tau)=\frac{3 Q}{\pi r_{o}^{2}} \exp \left\{-3\left(\frac{(x-r \cos \theta-v \tau)^{2}+\left(y-r \sin \theta-l_{\max } \cos \left(2 \pi \tau / \tau^{*}\right)\right)^{2}}{r_{o}^{2}}\right)\right\}
$$

where $l_{\max }$ is the amplitude and $\tau^{*}$ is the period of every cycle. For a rotating beam the beam intensity distribution is obtained as,

$$
\begin{gathered}
f(x-r \cos \theta, y-r \sin \theta, \tau)=\frac{3 Q}{\pi r_{o}^{2}} \times \exp \left\{-3\left(\frac{\left(x-r \cos \theta-v \tau-a \sin \left(2 \pi \tau / \tau^{*}\right)\right)^{2}}{r_{o}^{2}}\right.\right. \\
\left.\left.+\frac{+\left(y-r \sin \theta-l_{\max } \cos \left(2 \pi \tau / \tau^{*}\right)\right)^{2}}{2}\right)\right\}
\end{gathered}
$$


where $l_{\max }$ is the amplitude and $\tau^{*}$ is the period of every rotate and $a$ is the diameter of rotation along moving line, it should be chosen so that cover the area properly. The best choice for $a$ is half of the distance that laser in every period moves (i.e. $a=v \tau^{*} / 2$ ).

For a dithering beam with uniform velocity in every half of cycle the $f(x-r \cos \theta, y-r \sin \theta, \tau)$ changes and is obtained as,

$$
\begin{aligned}
& f(x-r \cos \theta, y-r \sin \theta, \tau)=
\end{aligned}
$$

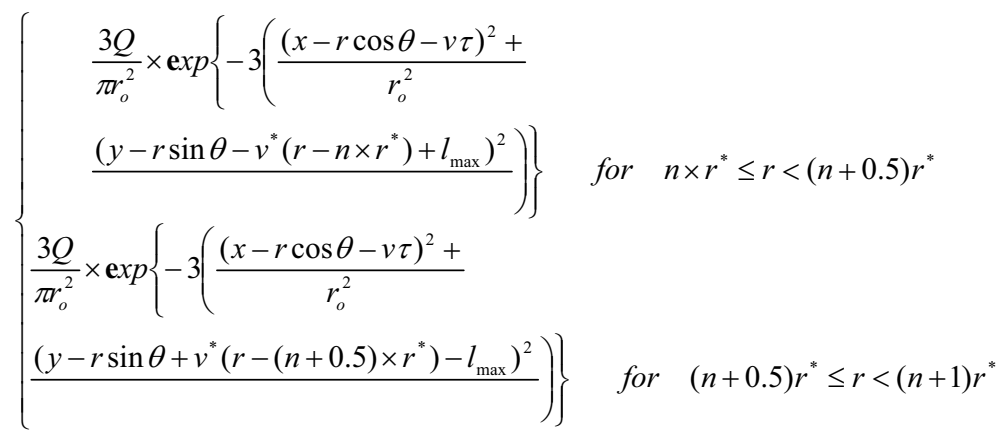

where $v$ is the laser velocity component along moving line (longitudinal velocity) and $v^{*}$ is the laser velocity component across moving line. For other beam intercity distributions $f\left(x^{\prime}, y^{\prime}, z^{\prime}, \tau\right)$ can be obtained (Farrahi \& Sistaninia, 2009).

\subsection{Numerical model}

The basic heat-transfer equations considered for the numerical model are,

$$
\mathrm{F}=\nabla(-K T)
$$

Which relates the heat flux $\mathbf{F}$ to thermal gradient

$$
\nabla(K \nabla T)-\rho c_{p} \frac{\partial T}{\partial t}=-G
$$

which applies to nonsteady-state heat conduction, where $G$ is the internal generation of heat. Internal heat generation, $G$, is zero for where the incident laser power is modeled as a heat flux. In this model, the time during the continuous laser irradiation is divided to $n$ time increments $\Delta t$. During each time increment $\Delta t$, it can be assumed that the laser beam does not move and the program determines the thermal load for each node on the heated surface, which depends on the laser beam position, heat intensity distribution and the node position. The laser beam position depends on the moving path, speed and time, thus the value of load for each node on the heated surface is a function of time and position. In each time increment, the finite element code ANSYS is used to compute the solution of Eq. (17). The result of every time increment is the initial condition of next time increment. For small $\Delta t$ it seems that the laser moves continuously. The flow chart of the finite element simulation is shown in 0 . 
In the model, it is necessary to make a decision about the element size and shape, time increment and number of step $\mathrm{n}^{*}$ for every time increment. This decision has been made with the help of the analytical model. Since these quantities are not independent, the following relations can be written.

$$
\left\{\begin{array}{l}
F_{o}=\frac{\Delta x^{2}}{\alpha \Delta t^{*}} \\
\Delta t^{*}=\frac{\Delta t}{n^{*}}
\end{array}\right.
$$

The Fourier number $F_{o}$, which includes material thermo-diffused coefficient $\alpha$, time step $\Delta t^{*}$ and node spacing $\Delta x$, should be below 2 . For small time increment every load step can be solved by one substep (i.e. $n^{*}=1$ and $\Delta t=\Delta t^{*}$ ).

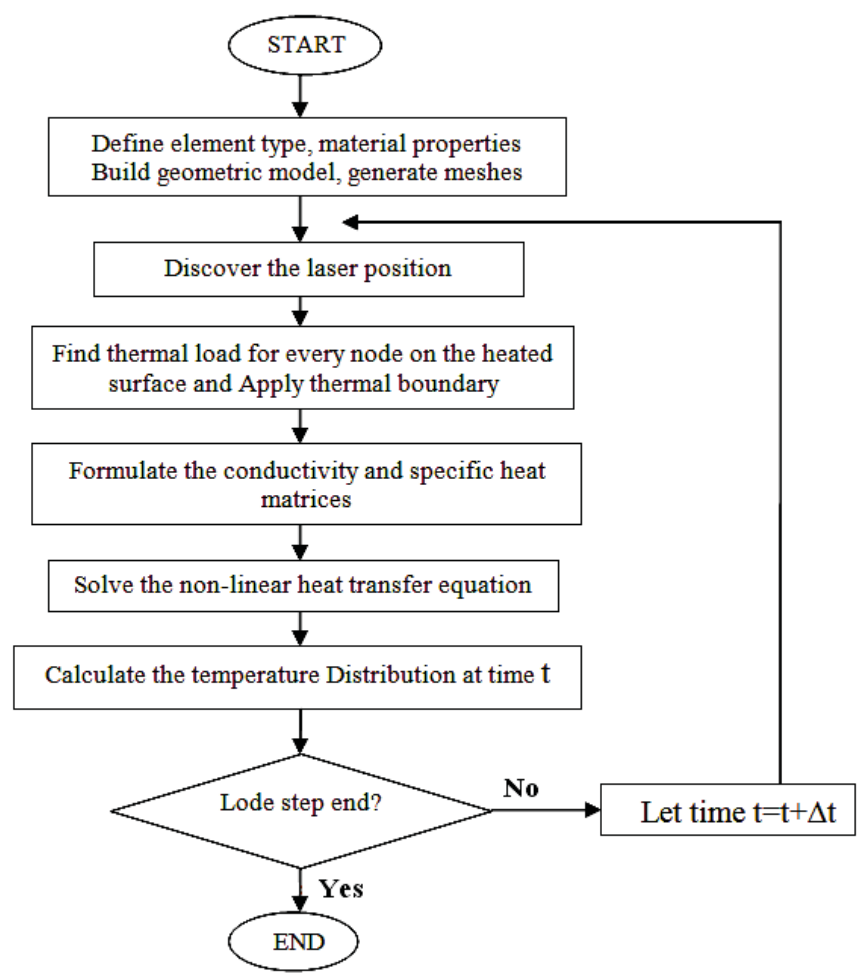

Fig. 3. Flow chart of the numerical thermal modeling

The effects of the elements size and time increment on the results of the finite element model can be studied by comparing the results of the finite element model with the results of the analytical model for uniform thermal properties. Figure 4 shows a comparison between the analytical results and the FEM results for different mesh sizes. This figure shows the 
temperature history of a point on the heated surface at $x=3.5 \mathrm{~mm}$ and $y=1 \mathrm{~mm}$ during LHD.

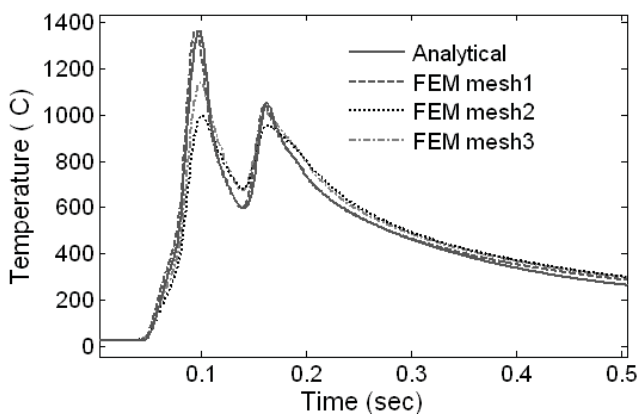

Fig. 4. Comparison of finite element model and analytical model results for LHD

In this comparison the model has been discretized by cubic element, in order to improve efficiency and reduce calculation, there are dense meshes around the heated region and for every cycle 12 load steps have been used. The thickness of the FEM model, $4 \mathrm{~mm}$ was chosen. The mesh 1 relates to a meshing for where the distance between nodes on the heated surface and around the heated region is $0.25 \mathrm{~mm}$, thus the mesh under the laser is such that it has sufficient number of elements to capture the inflection of the Gaussian distribution. As it is obvious for this case, there is a good agreement between FEM result and analytical result. The mesh 2 relates to a meshing which the distance between nodes on the heated surface and around the heated region is $0.5 \mathrm{~mm}$ and four elements were used along the zdirection (in thickness direction) and the mesh 3 relates to a meshing which the distance between the nodes on the heated surface and around the heated region again is $0.5 \mathrm{~mm}$ and eight elements were used along the z-direction.

Although the time of one cycle is so small $(0.1 \mathrm{~s})$, at least six load steps for every cycle are needed and if the number of the load steps for every cycle increase more than 12 load steps the error does not decrease significantly.

After that the parameters of the finite element model (elements sizes and time increments) are chosen properly, realistic effects like temperature-dependent thermal properties, specific heat and thermal conductivity, can be included in the model.

\section{Thermal analysis of laser hardening for different moving patterns}

In laser surface hardening, the work-piece is heated on the surface by a laser beam. The laser travels from one end of the work-piece to the other. In order to achieve the phase transformation, the temperature in the hardening zone, should be greater than the phase change temperature ( $\left.\mathrm{T}_{\text {phase }}\right)$. However, the temperature should not exceed the melting temperature and should be high and long for the austenitic transformation of steel to take place. In order to activate phase change, the cooling rate should also be greater than the critical cooling rate. The high cooling rate of the work-piece is achieved by the speed of the torch and the heat loss from the work-piece.

The models, are developed in the previous sections, is used in the analysis and optimization of the laser surface-hardening process of a gear tooth. Figure 7 is a schematic of the laser surface transformation hardening of a gear tooth with LHD, showing salient features of the 
process (Farrahi \& Sistaninia, 2009). In this work the laser beam traverses longitudinally along the work surface (x-axis) at a longitudinal velocity of $v_{\mathrm{sc}}$ followed by an appropriate transverses feed, $f$ along the y-axis. This sequence will be repeated till the entire surface of the gear tooth is hardened to a given depth. The dotted line indicates the required depth of hardening, which in this investigation is taken as $0.12 \mathrm{~mm}$. We adopt the same example (0) used In Ref. (Komanduri \& Hou, 2004).

The dimensions of the gear tooth used are shown in 0 , although only a $10 \mathrm{~mm}$ layer from heated surface are considered in order to reduce calculation time. A Laser beam of diameter $3 \mathrm{~mm}$, and normal intensity distribution (for LHD and LHR), power of $480 \mathrm{~W}$ and absorptivity of $A=71 \%$ is used. The gear is made of steel AISI 1036. The thickness of the hardening layer is taken as $0.12 \mathrm{~mm}$. The phase transition temperature for the AISI 1036 steel is taken as $775{ }^{\circ} \mathrm{C}$ and the melting temperature as $1470{ }^{\circ} \mathrm{C}$ and a critical martensite temperature of $430^{\circ} \mathrm{C}$ (Farrahi \& Sistaninia, 2009). In order to obtain Martensite, according to literatures, the martensizing temperature should be reached in less than $3.5 \mathrm{~s}$ from the austenizing temperature. Hence the critical cooling rate to achieve martensite is $91.43{ }^{\circ} \mathrm{C} / \mathrm{s}$. The material has non-linear conductivity $K$ and specific heat $c_{p}$, since these material properties depend on the temperature (ASM International, 1994). The diffusion process is a time dependent phenomenon; an interaction time of $15 \mathrm{~ms}$ is taken as a basis to determine the hardened region.

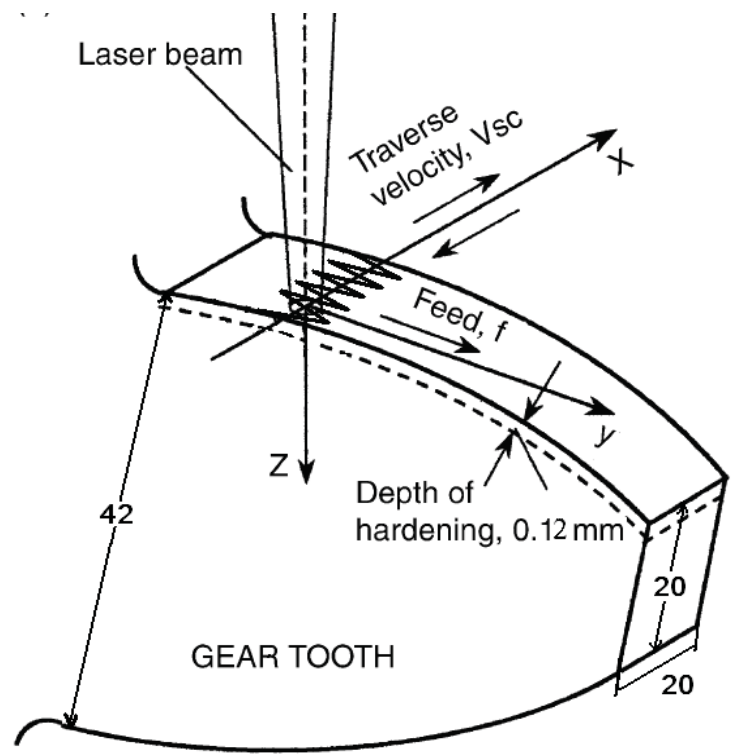

Fig. 5. Schematic of the laser surface transformation hardening of a gear tooth.

0 shows the variation of the maximum temperature in a section on the heated surface with the distance from the center line, $y$, for different moving patterns. The results of the LHD and LHR are compared with the laser hardening with, bimodal distribution $(5 \mathrm{~mm}$ of diameter) Ref. (Komanduri \& Hou, 2004) and rectangular Gaussian beam ( $w=3 \mathrm{~mm}$ and $r_{\mathrm{o}}=0.5 \mathrm{~mm}$ ) Ref. (Leung, 2001). For LHD with uniform velocity the maximum temperature 
takes place on the center line, and as the distance increases from the center line the temperature falls precipitously, and as a result of that the hardened depth changes across the hardened region and also maximum temperature is high so surface melting could occur.

(a)
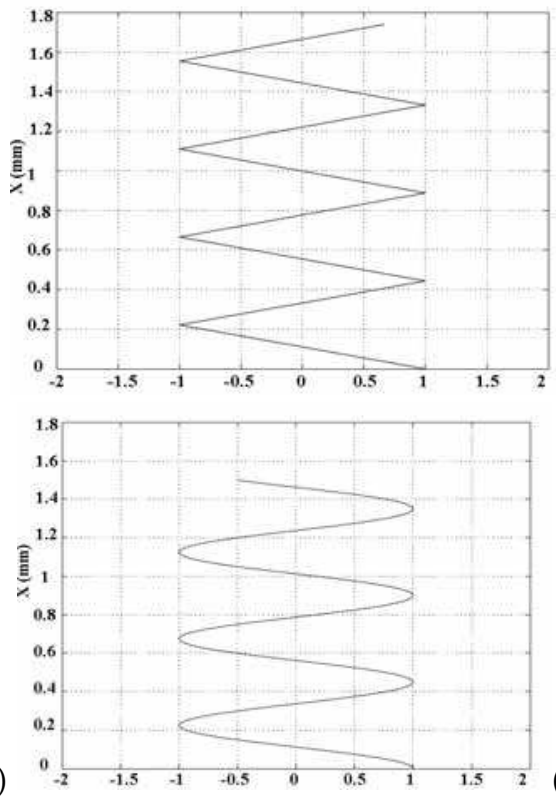

(b)

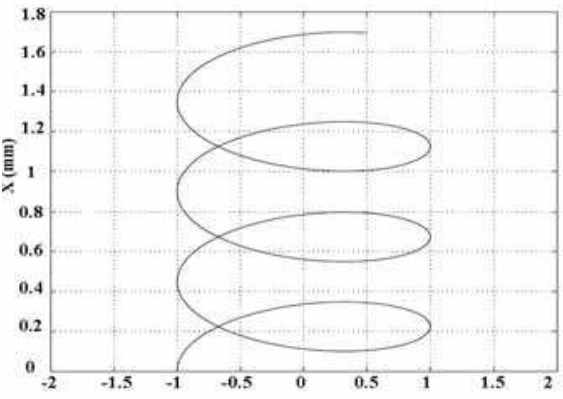

(d)

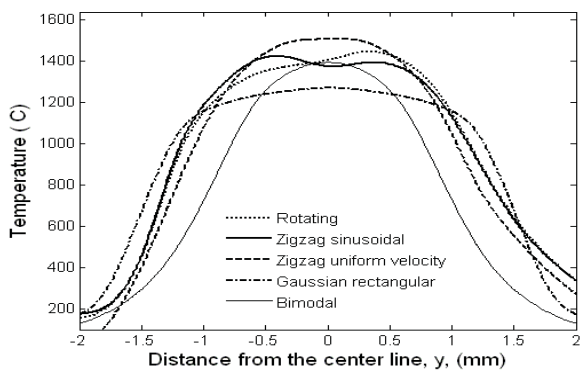

Fig. 6. (a) dithering beam with uniform velocity (b) rotating beam (c) dithering beam with sinusoidal velocities (d) Variation of the maximum temperature rise with the distance from the center line, $y$, for different moving patterns.

In similar case the maximum temperature of LHR is more than the maximum temperature of LHD with sinusoidal velocity and is less than the maximum temperature of LHD with uniform velocity. For laser hardening with LHD and LHR the cross feed (hardened bound) changes along the work surface ( $x$-axis). Because of that we have to determine maximum and minimum cross feed for every case. Maximum cross feed usually takes place on the cross section between two sequence peaks, for where the interaction time is enough for phase transformation.

Figure 7 shows the changes of maximum and minimum cross feed (for hardened depth of $0.12 \mathrm{~mm}$ ) with amplitude, for longitudinal velocities $0 \mathrm{f} 30 \mathrm{~mm} / \mathrm{sec}$, periods of 0.015 and for (a) dithering beam (b) rotating beam. 


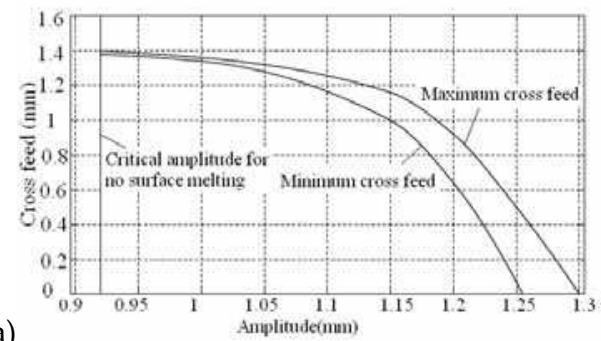

\section{(a)}

Fig. 7. Variations of cross feed with amplitude for (a) dithering Gaussian beam (b) rotating

(b)

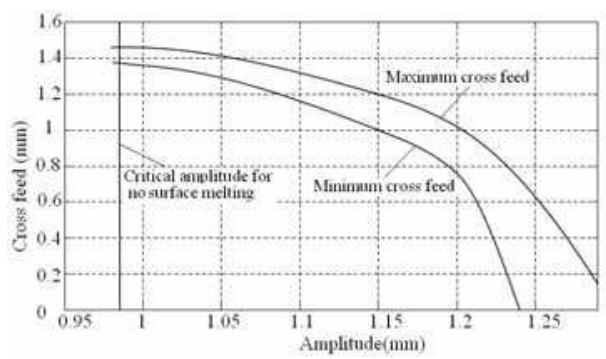

It can be seen that as the amplitudes decreases, the maximum temperature and cross feed increases until the maximum temperature reaches the melting temperature. As you see a similar trend of dithering beam is observed for rotating beam except that in similar case the critical amplitude for no surface melting is more than the amplitude for no surface melting in dithering beam, and also the difference between maximum and minimum cross feed is more than the difference between maximum and minimum cross feed of dithering beam.

\section{Laser forming of plates using rotating and dithering beams}

Laser forming is a novel technique, during that a laser beam causes thermal expansion locally, and deformation is achieved by scanning the laser beam across one side of the material. The temperature gradients developed through the material induce distortion because the temperature changes with thickness that causes different expansion of adjoining layers. Laser forming is currently used because of its technical benefits of, do not require external forces and thus reduces cost and increases flexibility.

\subsection{Laser forming analysis procedure}

The heat produced due to plastic deformation is small and can be ignored. Thermostructural problem can then be decoupled and solved sequentially in two steps; (i) transient thermal analysis, which has been done in section 2, (ii) structural analysis. In structural analysis the temperature distribution input as a body load to determine the residual stresses and distortions.

Ansys/explicit can be used to model both the initial load (thermal load), and to determine the final distortion. Ansys programming language (APDL) is used to model the laser beam motion scheme, and subsequent application of thermal loads in the structural analysis. Although, the structural analysis is not a transient analysis, since the nodal temperatures change with time the mechanical stresses and strains also change with time. However the inertia and damping forces need not be included, that is named quasi-static analysis.

For each load-step the result of the same load-step of the thermal model is used as the thermal load for that loadstep. Thus the numbers of the load-step for structural analysis are as many as the numbers of the load-step for thermal analysis. The flow chart of the structural simulation is shown in 0 . 


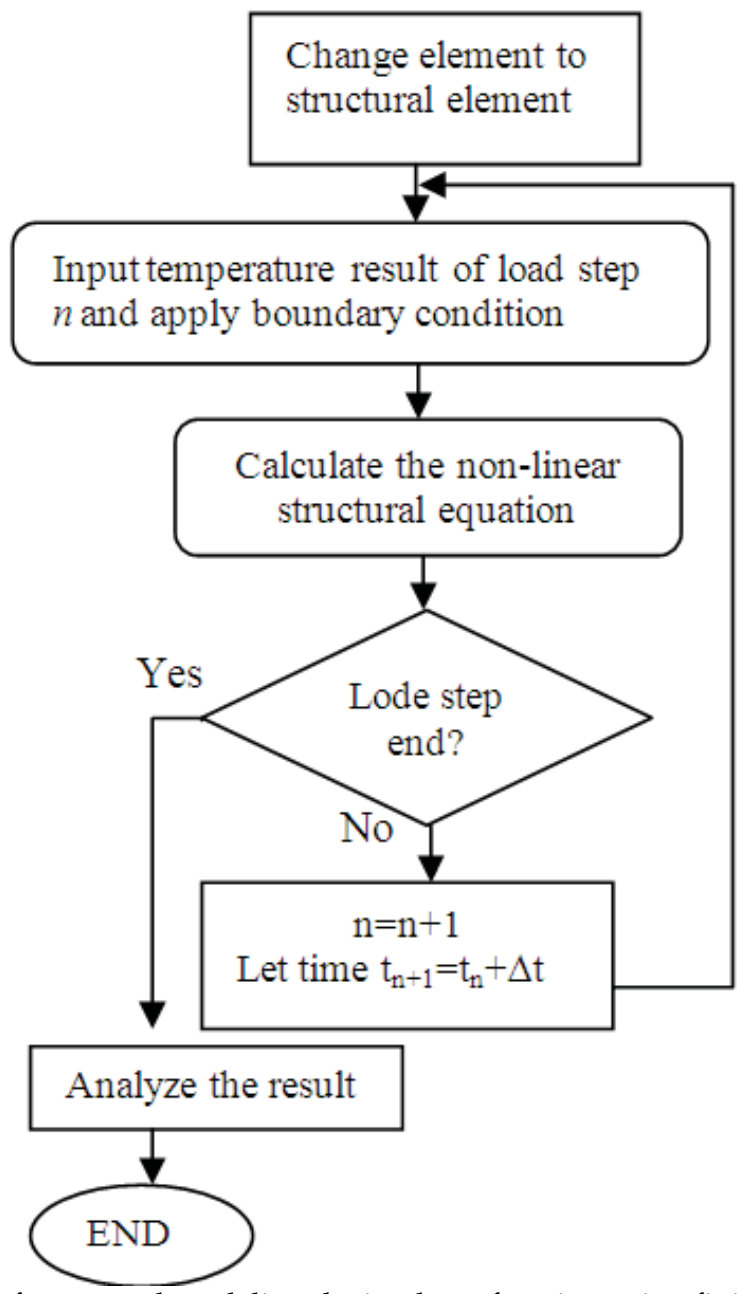

Fig. 8. Flow chart of structural modeling during laser forming using finite element method

Laser forming generates stresses exceeding yield stress within the specimen. The material subjected to the thermal cycle of the laser forming have to behave mechanically as an initially isotropic, elastoplastic and strain hardening continuum, so a component of total strain $\epsilon_{\mathrm{ij}}$ is given by the following formula,

$$
\epsilon_{i j}=\epsilon_{i j}+\epsilon p_{i j}+\epsilon^{t h}{ }_{i j}
$$

where $\epsilon^{e_{i j},} \in p_{i j}, \epsilon^{t h}{ }_{i j}$ are the component of elastic, plastic and thermal strain respectively. This behavior is modeled using a bilinear kinematic stress-strain curve (0) with strain-rate independence of the yield strength. Rate-independent plasticity is characterized by using the irreversible straining. 


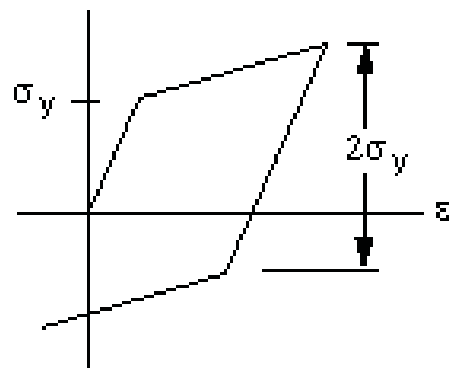

Fig. 9. Bilinear kinematic stress-strain curve

\subsection{Example problem}

The model, developed prior, is exemplified in the investigation of the laser forming of a stainless steel plate (AISI 304L). 0 shows a schematic of this process. A cubic of $30 \mathrm{~mm}$ along the direction of laser path and $30 \mathrm{~mm}$ vertical the direction of laser path and $2 \mathrm{~mm}$ thickness is used for this investigate. Temperature dependent physical and mechanical properties of the model are taken from Ref. (Safdar et al., 2007). The material is assumed to be homogenous and isotropic. The ambient temperature is set at $25{ }^{\circ} \mathrm{C}$.

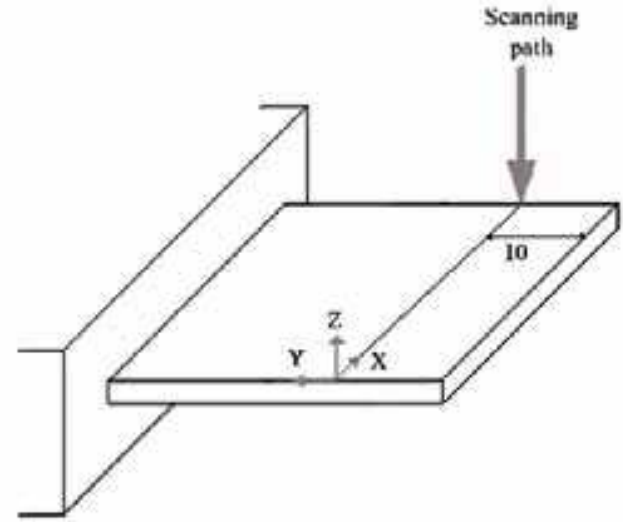

Fig. 10. Schematic of the laser forming of a plate

The following are the processing variables chosen for the laser heat treatment of the workpiece: laser power $500 \mathrm{~J} / \mathrm{s}$; absorptivity $\mathrm{A}=80 \%$, longitudinal velocity $30 \mathrm{~mm} / \mathrm{s}$ for rotating and dithering beam and $50 \mathrm{~mm} / \mathrm{s}$ for linear moving, beam diameter of $4 \mathrm{~mm}$, Gaussian beam distribution, period of $\tau^{*}=0.1 \mathrm{~s}$ and amplitude $l_{\max }=2 \mathrm{~mm}$.

0 shows the residual $\mathrm{Y}$ plastic strain contours and corresponding values on the heated surface (X, Y plane) and also on the $Y, Z$ plane for (a) dithering beam (b) rotating beam and (c) linear moving. As you see more plastic deformation can be developed by using rotating or dithering beam than using a beam with linear moving. 0 shows the temperature changes with time at various points on the heated surface at $x=15 \mathrm{~mm}$ for (a) dithering beam (b) rotating beam and (c) linear moving beam. During the initial heat-up the local severe temperature gradients in the vicinity of the scan path produces the compressive yielding in 
this region and near the heated surface. However, the thermal stress state changes to the tensile stress as the distance from the center line increases.

(a)
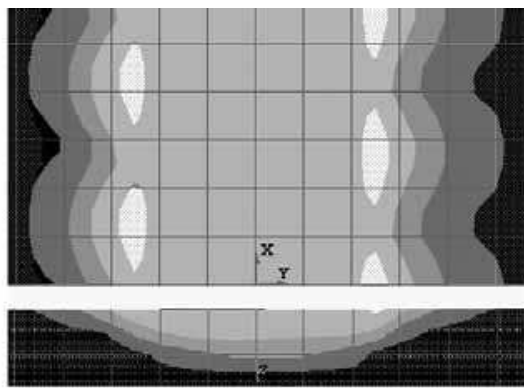

(b)
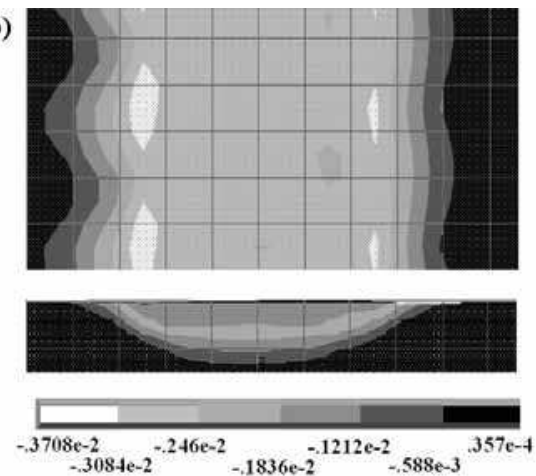

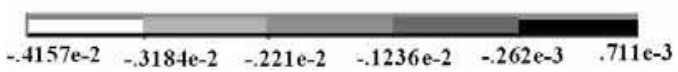

(c)
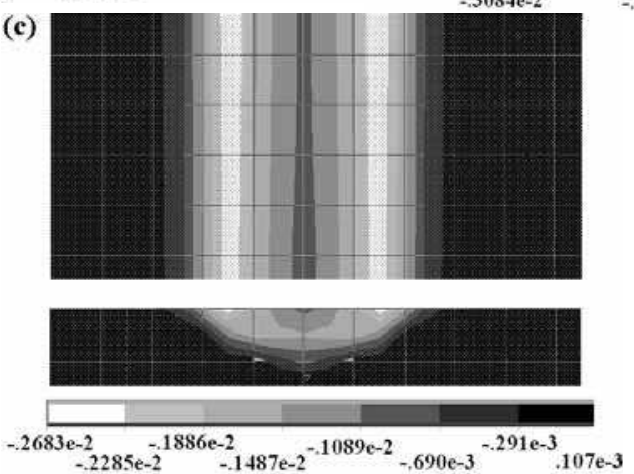

Fig. 11. Y plastic strain, contour and value using (a) dithering beam (b) rotating beam and (c) linear moving beam

(a)

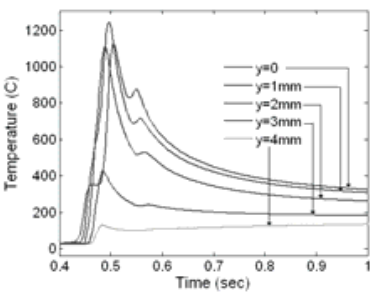

(b)

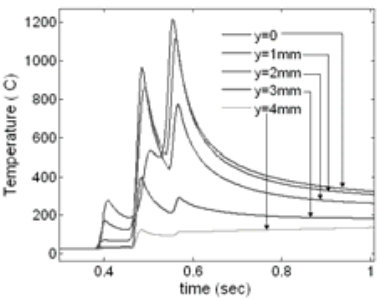

(c)

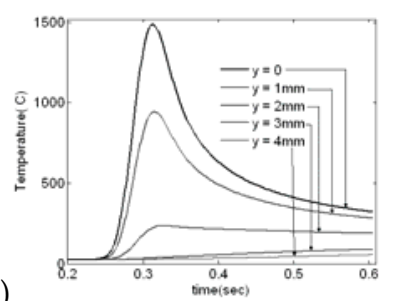

Fig. 12. Temperature history during laser forming using (a) dithering beam (b) rotating beam, and (c) linear moving beam

0 shows the displacement in Z-direction of the metal plate along the center line at $x=15$ $\mathrm{mm}$, during heating and cooling with (a) dithering beam (b) rotating beam and (c) linear moving beam. As you see in 0 upon the initial heat-up the different thermal expansion of the adjacent layers leads the free end of the plate to bend downward. As the temperature increases, the yield strength decreases rapidly, so the compressive thermal stress reduces in 
the vicinity of the scan path. During cooling, the yield strength increases and tension residual stresses is produced in the vicinity of the heated zoon. Hence, as the cooling proceeds, the portion of the specimen which is longitudinally in tension increases stably and the free end of the plate begins to bend upward. The plate also bends about Y-axis toward the laser beam because of the production of longitudinal tensile residual stress in the vicinity of the heated surface during cooling (Me Sistaninia et al., 2009).

(a)

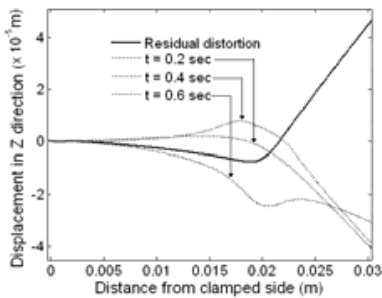

(b)

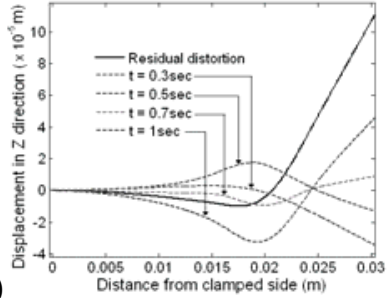

(c)

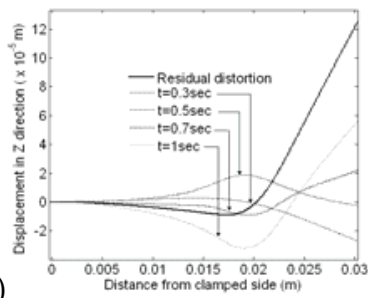

Fig. 13. Displacement in Z-direction of the metal plate along the center line at $x=15 \mathrm{~mm}$, during heating and cooling with (a) dithering beam (b) rotating beam and (c) linear moving beam.

It is obvious from 0 that the bending angel of the plate using LHD or LHR is 3 times more than the bending angel of laser forming using a beam which moves along a straight line with uniform velocity.

\section{Laser heat treatment considering coupled thermoelasticity}

The heat which is produced due to solid deformation is usually small and can be ignored. Because of that in a typical thermoelasticity formulation it is assumed that the thermal and mechanical fields are uncoupled in the material. Hence first the thermal fields are modeled and then the thermal results are used to find the displacement field and stress profile. In recent years, considerable researches have been carried out on the numerical analysis of coupled thermoelasticity problems e.g.(Bagri \& Eslami, 2008; Hosseini-Tehrani \& Eslami, 2000). Coupled thermoelasticity contains the phenomena which describe the elastic and thermal behavior of material. Many attempts have been made to solve the uncoupled thermoelasticity problems in steady or transient heat conduction states, but few investigations have been carried out successfully for coupled thermoelasticity problems. This section considers coupled thermoelasticity to find thermoelastic temperature, displacement and stress profile due to laser irradiation on the surface in both Eulerian and Lagrangian formulations.

Quasi-steady-state process is a process which is steady in either a stationary or a moving configuration. For uniform velocity and heat flux distribution, laser heat treatment with linear moving-beam leads to a quasi-steady-state process and the Eulerian formulation reduces to a steady-state problem. But, Lagrangian formulation for such process remains transient and need a transient analysis. The time-increment approach is used to solve transient problems numerically, with a great number of increments. Also, it is necessary to use dense meshes throughout the model length. Because of these reasons the run-time of Lagrangian analysis is much more than the run-time of Eulerian analysis. Therefore, the 
analysis of a quasi steady-state process using an Eulerian formulation is computationally efficient.

A semi infinite homogeneous, isotropic and thermoelastic solid is subjected to a moving Gaussian rectangular laser beam at $t>0$, as shown in Figure 1. Only a layer is considered which extends through $-b<y<b$ and $0<x<l$. The $l$ and $b$ are chosen long enough, such that it can be assumed that the initial conditions do not change on the boundary at $y=+b, y=-b$ and $x=0$. Thus, the layer is rigidly bonded along these bounds. The laser beam's energy is absorbed in the plane of the free surface and diffuses through the work-piece (Me. Sistaninia et al., 2009).
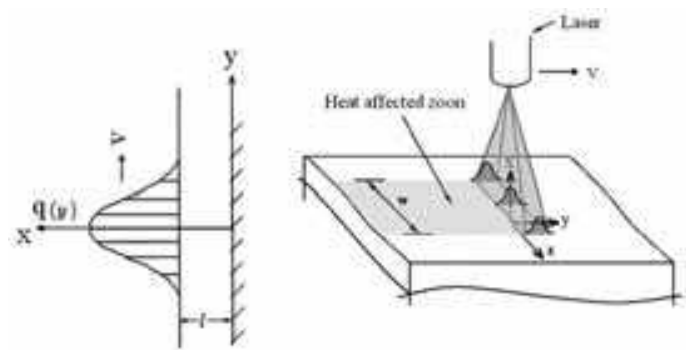

Fig. 14. Schematic of a material subjected to the thermal load from moving Gaussian rectangular laser beam

\section{1 Lagrangian formulation}

This section deals with the solution scheme for a transient problem using Lagrangian formulation. The governing equations for the dynamic coupled thermoelasticity in the absence of body forces can be written as follows (Hosseini-Tehrani \& Eslami, 2000):

$$
\begin{array}{r}
(\lambda+\mu) u_{j, i j}+\mu u_{i, j j}-\rho \ddot{u}_{i}-\gamma T_{, i}=0 \\
k T_{, i i}-\rho c \dot{T}-\gamma T_{o} \dot{u}_{j, j}=0
\end{array}
$$

where $u_{i}(\mathrm{i}=1,2,3)$ denote the components of displacement vector; $\lambda, \mu$ the Lame's constants; $\rho$ the density, $T$ the absolute temperature, $T_{o}$ the reference temperature, $\gamma$ the stresstemperature modulus, $k$ the conductivity and $c$ denotes the specific heat at constant strain or volume which is equal to:

$$
c=c_{p}-3 \frac{T_{o}}{\rho} \alpha_{L} \gamma
$$

where $c_{p}$ is specific heat at constant stress or pressure (notice that for uncoupled thermoelasticity $c=c_{p}$ ) and $\alpha_{L}$ is linear thermal expansion coefficient. In Eqs. (1) and (2) the superscript (.) denotes the time differentiation (e.g. $\dot{u}=\frac{d u}{d t}$ and $\ddot{u}=\frac{d^{2} u}{d t^{2}}$ ) and the subscript (,) denotes the partial differentiation with respect to the space variables. 
Notice that in a typical Lagrangian system, the work-piece configuration is fixed in space and the reference configuration is fixed to the material and all the spatial parameters are calculated with respect to this reference configuration.

It is convenient to introduce the dimensionless variables as follows:

$$
\begin{aligned}
& \hat{x}=\frac{x}{v}, \quad \hat{t}=\frac{t C_{s}}{v}, \quad \hat{\sigma}_{i j}=\frac{\sigma_{i j}}{\gamma T_{o}}, \\
& \hat{u}_{i}=\frac{(\lambda+2 \mu) u_{i}}{v \gamma T_{o}}, \quad \hat{T}=\frac{T-T_{o}}{T_{o}},
\end{aligned}
$$

where $C_{s}=\sqrt{(\lambda+2 \mu) / \rho}$ is the velocity of propagation of the longitudinal wave and $v=k / \rho c C_{s}$ is the dimensionless unit length. For two-dimensional domain, after using the dimensionless variables (22), the equations (20-a) and (20-b) reduce to;

$$
\left\{\begin{array}{c}
\left(\frac{\bar{\mu}}{(\bar{\lambda}+2 \mu)} \Delta+\frac{(\bar{\lambda}+\mu)}{(\bar{\lambda}+2 \mu)} D_{1}^{2}\right) \hat{u}_{1}+\left(\frac{(\bar{\lambda}+\mu)}{(\bar{\lambda}+2 \mu)} D_{1} D_{2}\right) \hat{u}_{2}-\ddot{\hat{u}}_{1}-D_{1} \hat{T}=0 \\
\left(\frac{(\bar{\lambda}+\mu)}{(\bar{\lambda}+2 \mu)} D_{1} D_{2}\right) \hat{u}_{1}+\left(\frac{\mu}{(\bar{\lambda}+2 \mu)} \Delta+\frac{(\bar{\lambda}+\mu)}{(\bar{\lambda}+2 \mu)} D_{2}^{2}\right) \hat{u}_{2}-\ddot{\hat{u}}_{2}-D_{2} \hat{T}=0 \\
-\left(C D_{1}\right) \dot{\hat{u}}_{1}-\left(C D_{2}\right) \dot{\hat{u}}_{2}+\Delta \hat{T}-\hat{\hat{T}}=0
\end{array}\right.
$$

Where $D_{i}=\partial / \partial \hat{x}_{i} \quad(\mathrm{i}=1,2), \Delta$ denote the Laplacian and $C=\frac{T_{o} \gamma^{2}}{\rho c(\lambda+2 \mu)}$ is coupling parameter. Under plane strain assumptions, $\bar{\lambda}$ and $\bar{\gamma}$ can be related to $\lambda, \mu$ and stresstemperature modulus $\gamma=(3 \lambda+2 \mu) \alpha_{L}$ as follows:

$$
\bar{\lambda}=\lambda \quad \text { and } \quad \bar{\gamma}=\gamma
$$

A transient finite element model is used to solve Eq. (23). Applying the variational principle produces the following finite element matrix equation (ANSYS Theory Manual, 2008):

$$
\left[\begin{array}{cc}
{[M]} & {[0]} \\
{[0]} & {[0]}
\end{array}\right]\left\{\left\{\begin{array}{l}
\{\ddot{\hat{u}}\} \\
\dot{\hat{T}}
\end{array}\right\}+\left[\begin{array}{cc}
{[0]} & {[0]} \\
{\left[C^{t u}\right]} & {\left[C^{t}\right]}
\end{array}\right]\left\{\begin{array}{l}
\{\dot{\hat{u}}\} \\
\{\dot{\hat{T}}\}
\end{array}\right\}+\left[\begin{array}{cc}
{[K]} & {\left[K^{u t}\right]} \\
{[0]} & {\left[K^{t}\right]}
\end{array}\right]\left\{\begin{array}{l}
\{\hat{u}\} \\
\{\hat{T}\}
\end{array}\right\}=\left\{\begin{array}{l}
\{F\} \\
\{Q\}
\end{array}\right\}\right.
$$

Finite element code Ansys can be used to solve the Eq. (25). For this purpose, a special 8node element called PLANE 223 Element in ANSYS guide should be used to discretize the geometry. The PLANE 223 Element is a 2-D coupled-field solid with up to four degrees of freedom per node. The thermal and mechanical boundary conditions are, 


$$
\begin{aligned}
& T=0 \quad \text { at } \quad t=0 \\
& T=0 \quad \text { at } \quad x=0 \\
& T=0 \quad \text { at } \quad y= \pm b \\
& u_{1}=u_{2}=0 \quad \text { at } \quad x=0 \\
& u_{1}=u_{2}=0 \quad \text { at } \quad y= \pm b \\
& \sigma_{x}=\sigma_{x y}=0 \quad \text { at } \quad x=l \\
& q(y, t)=\frac{3 Q}{w \sigma^{\prime}} \exp \left\{-3\left(\frac{y-v t}{r_{o}}\right)^{2}\right\} \quad \text { at } \quad x=l
\end{aligned}
$$

where $w$ is the length of the Gaussian rectangular heat source and $r_{o}$ is the characteristic radius.

\section{2 Eulerian formulation}

As mentioned, in Eulerian formulation, the problem is considered as a steady-state problem. In Eulerian formulation the reference configuration is a control volume through that the work-piece moves. The derivative of variables (temperature and displacements) in the moving body with respect to time are given by,

$$
\frac{\mathrm{d} U_{i}}{\mathrm{~d} t}=\frac{\partial U_{i}}{\partial t}+\frac{\partial U_{i}}{\partial x_{j}} \frac{\mathrm{d} x_{j}}{\mathrm{~d} t}, \quad \mathrm{i}=1,2,3
$$

where $U_{i}=\left[\begin{array}{lll}u_{1} & u_{2}\end{array}\right]$ and $x_{j}(j=1,2)$ is the material system coordinate which moves with a velocity $\mathbf{v}$ in space. Hence Eq. (27) can be expressed as,

$$
\frac{\mathrm{d} U_{i}}{\mathrm{~d} t}=\frac{\partial U_{i}}{\partial t}+\mathbf{v} \cdot \nabla U_{i}
$$

Also, the second derivative of displacements in the moving body with respect to time is given by,

$$
\frac{\mathrm{d}^{2} u_{i}}{\mathrm{~d} t^{2}}=\frac{\partial V_{i}}{\partial t}+\mathbf{v} \cdot \nabla V_{i}, \quad \mathrm{i}=1,2
$$

where $V_{i}=\left[\begin{array}{ll}\frac{\mathrm{d} u_{1}}{\mathrm{~d} t} & \frac{\mathrm{d} u_{2}}{\mathrm{~d} t}\end{array}\right]$ and $\nabla=\mathrm{e}_{\mathrm{m}} \frac{\partial}{\partial x_{m}}, \mathrm{~m}=1,2$.

Since the control volume has a steady-state temperature and displacement profile, for a quasi-steady-state process the Eqs. (28) and (29) reduce to

$$
\frac{\mathrm{d} U_{i}}{\mathrm{~d} t}=\mathrm{v} D_{2} U_{i}, \quad \mathrm{i}=1,2,3
$$




$$
\frac{\mathrm{d}^{2} u_{i}}{\mathrm{~d} t^{2}}=\mathrm{v}^{2} D_{2}^{2} u_{i}, \quad \mathrm{i}=1,2
$$

Hence the Eq. (23) in Eulerian formulation reduces to (Me. Sistaninia et al., 2009),

$$
\left\{\begin{array}{l}
\left(\frac{\mu}{(\lambda+2 \mu)} \Delta+\frac{(\lambda+\mu)}{(\lambda+2 \mu)} D_{1}^{2}\right) \hat{u}_{1}+\left(\frac{(\lambda+\mu)}{(\lambda+2 \mu)} D_{1} D_{2}\right) \hat{u}_{2}-D_{1} \hat{T}=0 \\
\left(\frac{(\lambda+\mu)}{(\lambda+2 \mu)} D_{1} D_{2}\right) \hat{u}_{1}+\left(\frac{\mu}{(\lambda+2 \mu)} \Delta+\frac{(\lambda+\mu)}{(\lambda+2 \mu)} D_{2}^{2}\right) \hat{u}_{2}-D_{2} \hat{T}=0 \\
-\left(C U^{*} D_{1} D_{2}\right) \hat{u}_{1}-\left(C U^{*} D_{2}^{2}\right) \hat{u}_{2}+\left(\Delta-U^{*} D_{2}\right) \hat{T}=0
\end{array}\right.
$$

where $U^{*}=\mathrm{v} / C_{s}$ is the dimensionless velocity. The production of the coupling parameter and dimensionless velocity can be called effective coupling parameter, $C_{e}=C U^{*}$. The boundary conditions in the Eulerian formulation are similar to those in the Lagrangian formulation. The boundary condition of the heat flux in Eulerian configuration takes the following form at $x=l$,

$$
\mathrm{q}(\mathrm{y})=\frac{3 Q}{w \sigma^{\prime}} \exp \left\{-3\left(\frac{y}{\sigma^{\prime}}\right)^{2}\right\}
$$

Finite element method is chosen as a convenient method for solving the equations of the Eulerian formulation. The geometry of the work-piece is discretized into a number of 6node triangle elements and the Galerkin finite element technique is used to obtain the solution. The displacements $\left(\hat{u}_{1}^{(e)}, \hat{u}_{2}^{(e)}\right)$ and temperature $\left(\hat{T}^{(e)}\right)$ are approximated as,

$$
\hat{u}_{1}^{(e)}=\sum_{i=1}^{6} N_{i} \hat{u}_{i}^{*}, \quad \hat{u}_{2}^{(e)}=\sum_{i=1}^{6} N_{i} \hat{v}_{i}^{*}, \quad \hat{T}^{(e)}=\sum_{i=1}^{6} N_{i} \hat{T}_{i}^{*}
$$

Here, $N_{i}$ is the shape function approximating the displacement and temperature fields in the element (e). Terms, $\hat{u}_{i}^{*}, \hat{v}_{i}^{*}$ and $\hat{T}_{i}^{*}$ define the nodal values of displacements and temperature, respectively. Using Eq. (34) and applying the Galerkin finite element method finally, for Eulerian formulation, the finite element matrix Eq. (25) reduce to (Me. Sistaninia et al., 2009),

$$
[\mathrm{K}]]\left\{\begin{array}{l}
\left\{\hat{\mathrm{u}}_{1}\right\} \\
\left\{\hat{\mathrm{u}}_{2}\right\} \\
\{\hat{\mathrm{T}}\}
\end{array}\right\}=\left\{\begin{array}{l}
\left\{\mathrm{F}_{\mathrm{x}}\right\} \\
\left\{\mathrm{F}_{\mathrm{y}}\right\} \\
\{\mathrm{Q}\}
\end{array}\right\}
$$

where, 
Where,

$$
[\mathrm{K}]=\left[\begin{array}{lll}
{\left[k^{u u}\right]} & {\left[k^{u v}\right]} & {\left[k^{u T}\right]} \\
{\left[k^{v u}\right]} & {\left[k^{v v}\right]} & {\left[k^{v T}\right]} \\
{\left[k^{T u}\right]} & {\left[k^{T v}\right]} & {\left[k^{T T}\right]}
\end{array}\right]
$$

$$
\begin{array}{r}
k_{i j}^{u u}=\int_{A^{(e)}}\left[\frac{\partial N_{i}}{\partial x} \frac{\partial N_{j}}{\partial x}+\frac{\mu}{(\lambda+2 \mu)} \frac{\partial N_{i}}{\partial y} \frac{\partial N_{j}}{\partial y}\right] d A \\
k_{i j}^{u v}=\int_{A^{(e)}}\left[\frac{\lambda}{(\lambda+2 \mu)} \frac{\partial N_{i}}{\partial x} \frac{\partial N_{j}}{\partial y}+\frac{\mu}{(\lambda+2 \mu)} \frac{\partial N_{i}}{\partial y} \frac{\partial N_{j}}{\partial x}\right] d A \\
k_{i j}^{v u}=\int_{A^{(e)}}\left[\frac{\lambda}{(\lambda+2 \mu)} \frac{\partial N_{i}^{u T}}{\partial y} \frac{\partial N_{j}}{\partial x}+\frac{\mu}{(\lambda+2 \mu)} \frac{\partial N_{i}}{\partial x} \frac{\partial N_{j}}{\partial x} \frac{\partial N_{j}}{\partial y}\right] d A \\
k_{i j}^{v v}=\int_{A^{(e)}}\left[\frac{\partial N_{i}}{\partial y} \frac{\partial N_{j}}{\partial y}+\frac{\mu}{(\lambda+2 \mu)} \frac{\partial N_{i}}{\partial x} \frac{\partial N_{j}}{\partial x}\right] d A \\
k_{i j}^{v T}=\int_{A^{(e)}}\left[-\frac{\partial N_{i}}{\partial y}\right] d A \\
k_{i j}^{T T}=\int_{A^{(e)}}\left[-U^{*} \frac{\partial N_{i}}{\partial y} N_{j}+\frac{\partial N_{i}}{\partial y} \frac{\partial N_{j}}{\partial y}+\frac{\partial N_{i}}{\partial x} \frac{\partial N_{j}}{\partial x}\right] d A \\
k_{i j}^{T\left(T^{(e)}\right.}=\int_{A^{(e)}}\left[-C U^{*} \frac{\partial N_{i}}{\partial y} \frac{\partial N_{j}}{\partial x}\right] d A \\
\left.-C U^{*} \frac{\partial N_{i}}{\partial y} \frac{\partial N_{j}}{\partial y}\right] d A
\end{array}
$$

For the elements on the heated surface $\left\{F_{x}\right\}=\left\{F_{y}\right\}=0$, and

$$
\{\mathrm{Q}\}=\mathrm{Q}_{\mathrm{i}}=\int_{\Gamma^{(e)}} \frac{3 Q \alpha_{L}}{w r_{o} T_{o} k} \exp \left(-3\left(\frac{y \alpha_{L}}{r_{o}}\right)^{2}\right) N_{i} d y=0
$$

where $N_{i}$ are the shape functions of the nodes placed on the heated surface.

\section{3 Numerical example}

The 30-mm-long and 10-mm-thick rectangular work-piece of AISI 1045 steel shown in Figure 1 has been used for this numerical example. Laser processing parameters used are $Q=450 \mathrm{~W}, w=10 \mathrm{~mm}$ and $r_{o}=1.5 \mathrm{~mm}$. The thermal field of this heat intensity distribution can be analyzed as a two dimensional heat conduction problem (Me. Sistaninia et al., 2009). 
Figures 3 and 4 show the comparison of the dimensionless variables changes along $Y$ direction on the heated surface. Figure 3 shows the temperature changes and Figure 4 shows the changes of the displacement in $\mathrm{X}$ direction. The results are plotted for effective coupling parameters of $C e=0$ and $C e=1 e-5$. Although the $C e$ at temperatures bigger than $T_{\text {phase }}$ because of phase transformation, is much bigger than $C e=1 e-5$, this value is unrealistically high for the metals at room temperature. The case of $C e=0$ corresponds to the uncoupled solution.

As shown in 0, the Eulerian formulation results are in good agreement with the Lagrangian formulation results. Also the thermal results of Eulerian and Lagrangian formulations, as shown in Figure 3, agree well with the analytical result in the case of the uncoupled solution $(C e=0)$. Although the results of the Eulerian and Lagrangian formulations are quite similar but, the run-times for two analyses differ very much. The Lagrangian formulation takes much more time to fulfill the analysis.

0 shows that the coupled temperature and displacement are more than the temperature and displacement which are obtained from uncoupled theory $(\mathrm{Ce}=0)$. So the coupling acts as a power generator in the system. The volume change of the austenitic transformation which occurs during laser hardening is large in comparison with the normal thermal expansion. The expansion leads to a thermal pressure shock in the work-piece. The pressure shock induces an extra temperature which causes the predicted temperature by coupled thermoelasticity to be more than the temperature predicted by uncoupled thermoelasticity. The mathematical reason may be followed considering Eqs. (5) and (6). The large volume change leads to a high coupling parameter for laser hardening, causing a considerable difference between the coupled and uncoupled thermoelsticity results.

(a)

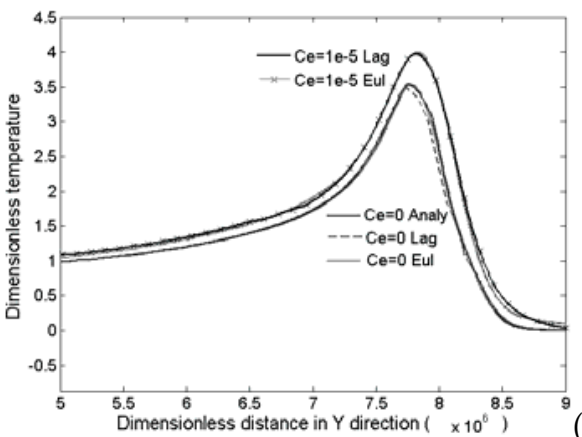

(b)

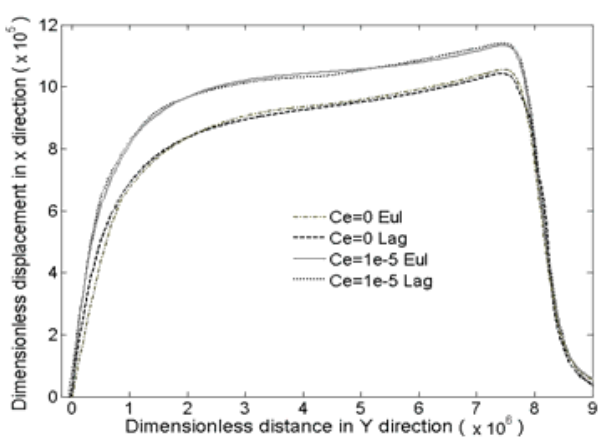

Fig. 15. (a) Comparison of the dimensionless temperature on the heated surface (b) Comparison of the dimensionless displacement in X direction on the heated surface

0 shows the contours of the dimensionless stress in $\mathrm{X}$ direction, $\mathrm{S}_{\mathrm{x}}$, and the corresponding values. As it is expected the $S_{x}$ on the heated surface is zero and under the heated surface, near the heated region, is tensile. During the heat-up, the local severe temperature gradients in the vicinity of the heat affected zoon causes a large expansion of the material in this region; but this material is constrained along $\mathrm{Y}$ direction (radically) by the surrounding material. The constraint leads to compressive stresses in $\mathrm{Y}$ directions in this region and near the heated surface which exceed yield stress within the target material. The thermal stresses 
state changes to tensile stress as the distance from the heated surface increases. The compressive stress in $\mathrm{Y}$ direction causes tensile stress in $\mathrm{X}$ direction.
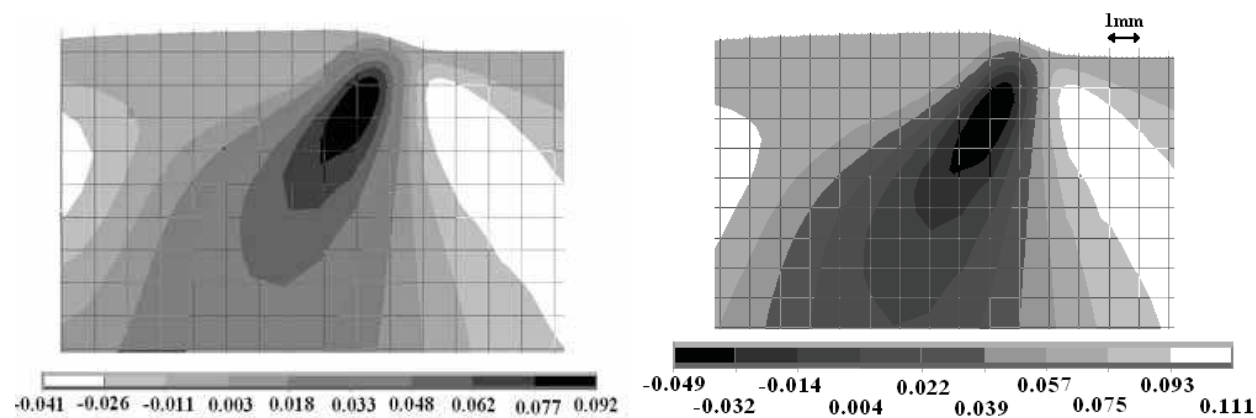

Fig. 16. Contours of the dimensionless stress, $\mathrm{Sx}$ for the effective coupling parameters (a) $C e=0$ (b) $C e=1 e-5$

\section{Conclusion}

The transient thermal fields during LHD and LHR were modeled by employing both threedimensional analytical model and finite element model. Then the transient thermal fields were analyzed to predict the hardened region of laser hardening of a medium carbon steel. The results show that using rotating and dithering beams is a useful method for laser hardening of metals when the parameters of dithering or rotating are chosen well by implementing these models. The temperature distribution from the thermal model was also inputted as a body load in a three-dimensional nonlinear structural analysis to determine the bending angle and distortions in laser forming with dithering and rotating beams. Thermoelastic temperature, displacement and stress in heat transfer during laser surface hardening were also solved in both Lagrangian and Eulerian formulations of coupled thermoelectricity. The simulations and observations of the deformations and temperatures lead to the following conclusions:

- For appropriate FEM parameters a good agreement between the result of FEM and the result of the analytical model obtains

- Dithering or rotating beam is a useful way to spreading out a laser beam, in order to obtain a larger and more uniform depth heat affected zoon,

- The bending angel of laser forming using rotating and dithering beams in similar conditions is several times (three times for the case investigated in this work) more than the bending angel of laser forming using a beam which moves along a straight line with uniform velocity

- The coupled temperature and displacement are more than the temperature and displacement which are obtained from uncoupled theory $(\mathrm{Ce}=0)$, hence the coupling acts as a power generator in the system 


\section{References}

ANSYS Theory Manual. (2008). Structure with material non linearities and coupled field analysis guide, ANSYS Inc., USA.

ASM International. (1994). ASM Handbook: properties and selection, vol. 1, ASM International,.

Bagri, A., \& Eslami, M. R. (2008). Generalized coupled thermoelasticity of functionally graded annular disk considering the Lord-Shulman theory. Composite Structures, Vol. 83, No. 2, (2008), pp. 168-179.

Farrahi, G. H., \& Sistaninia, M. (2009). Thermal analysis of laser hardening for different moving patterns. International journal of engineering, Vol. 22, No. 2, (2009), pp. $169-180$

Hosseini-Tehrani, P., \& Eslami, M. R. (2000). BEM analysis of thermal and mechanical shock in a two-dimensional finite domain considering coupled thermoelasticity. Engineering Analysis with Boundary Elements, Vol. 24, No. 3, (2000), pp. 249-257.

Komanduri, R., \& Hou, Z. B. (2004). Thermal analysis of laser surface transformation hardening--optimization of process parameters. International Journal of Machine Tools and Manufacture, Vol. 44, No. 9, (2004), pp. 991-1008.

Leung, M. (2001). Phase-change heat transfer in laser transformation hardening by moving Gaussian rectangular heat source. Journal of Physics D: Applied Physics, Vol. 34, No. 24, (2001), pp. 3434-3441.

Luxon, J. (1984). Laser - Industrial Applications, Prentice Hall, New Jersey.

Polyanin, A. D. (2002). Handbook of Linear Partial Differential Equations for Engineers and Scientists, Chapman \& Hall/CRC,1584882999, Georgia.

Rajadhyaksha, S. M., \& Michaleris, P. (2000). Optimization of thermal processes using an Eulerian formulation and application in laser surface hardening. Int. J. Numer. Meth. Engng, Vol. 47, No. 11, (2000), pp. 1807-1823.

Safdar, S., Li, L., Sheikh, M. A., \& Zhu, L. (2007). Finite element simulation of laser tube bending: Effect of scanning schemes on bending angle, distortions and stress distribution. Optics \& Laser Technology, Vol. 39, No. 6, (2007), pp. 1101-1110.

Sistaninia, Me., Sistaninia, Ma., \& Moeanodini, H. (2009). Laser forming of plates using rotating and dithering beams. Computational Materials Science, Vol. 45, No. 2, (2009), pp. 480-488.

Sistaninia, Me., Sistaninia, Ma., \& Moeanodini, H. (2009). Laser surface hardening considering coupled thermoelasticity. Journal of mechanic, Vol. 25, No. 3, (2009), pp. $241-249$ 


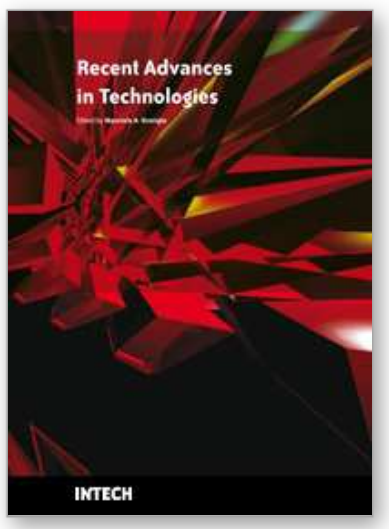

\author{
Recent Advances in Technologies \\ Edited by Maurizio A Strangio
}

ISBN 978-953-307-017-9

Hard cover, 636 pages

Publisher InTech

Published online 01, November, 2009

Published in print edition November, 2009

The techniques of computer modelling and simulation are increasingly important in many fields of science since they allow quantitative examination and evaluation of the most complex hypothesis. Furthermore, by taking advantage of the enormous amount of computational resources available on modern computers scientists are able to suggest scenarios and results that are more significant than ever. This book brings together recent work describing novel and advanced modelling and analysis techniques applied to many different research areas.

\title{
How to reference
}

In order to correctly reference this scholarly work, feel free to copy and paste the following:

M. Sistaninia (2009). Laser Heat Treatment of Metals Using Rotating and Dithering Beams, Recent Advances in Technologies, Maurizio A Strangio (Ed.), ISBN: 978-953-307-017-9, InTech, Available from: http://www.intechopen.com/books/recent-advances-in-technologies/laser-heat-treatment-of-metals-usingrotating-and-dithering-beams

\section{INTECH}

open science | open minds

\section{InTech Europe}

University Campus STeP Ri

Slavka Krautzeka 83/A

51000 Rijeka, Croatia

Phone: +385 (51) 770447

Fax: +385 (51) 686166

www.intechopen.com

\section{InTech China}

Unit 405, Office Block, Hotel Equatorial Shanghai

No.65, Yan An Road (West), Shanghai, 200040, China

中国上海市延安西路65号上海国际贵都大饭店办公楼405单元

Phone: +86-21-62489820

Fax: $+86-21-62489821$ 
(C) 2009 The Author(s). Licensee IntechOpen. This chapter is distributed under the terms of the Creative Commons Attribution-NonCommercial-ShareAlike-3.0 License, which permits use, distribution and reproduction for non-commercial purposes, provided the original is properly cited and derivative works building on this content are distributed under the same license. 